\title{
Autodeterminación de las personas con discapacidad intelectual como objetivo educativo y derecho básico: estado de la cuestión
}

\author{
Self-determination of individuals with \\ intellectual disability as an educational goal \\ and basic right: The status of the issue
}

\section{Resumen}

Los resultados de la investigación sugieren que la autodeterminación es una de las variables que determina, en parte, la calidad de vida de las personas con discapacidad intelectual y su reconocimiento como miembros de pleno derecho. Este artículo presenta una síntesis de las principales ideas acerca de este constructo: factores que posibilitan el origen y desarrollo de este movimiento, modelos conceptuales, instrumentos de evaluación y sugerencias para la intervención. Para ello, se ha realizado una exhaustiva revisión de la literatura más significativa al respecto. Los esfuerzos dirigidos a promover este derecho básico nos permiten afirmar que la autodeterminación ha dejado de ser un deseo para pasar a ser una realidad.

\section{Palabras clave}

Autodeterminación, discapacidad intelectual, intervención educativa, revisión.

\begin{abstract}
Results of research suggest that selfdetermination is one of the variables that determine, in part, the quality of life of people with intellectual disabilities and their recognition as equal members of society. This paper presents a summary of the main ideas about this construct: factors that enable the origin and development of this movement, conceptual models, assessment tools and suggestions for intervention. To do this, we have performed an exhaustive review of the literature. Efforts to promote this basic right let us to state that selfdetermination is no longer a wish but it has become a reality.
\end{abstract}

\section{Keywords}

Self-determination, intellectual disabilities, educational intervention, review.

\section{Araceli Arellano Torres <aarellanot@unav.es> \\ Instituto de Cultura y Sociedad, Departamento de Educación. Universidad de Navarra}

\section{Feli Peralta López}

Instituto de Cultura y Sociedad, Departamento de Educación. Universidad de Navarra

\author{
Para citar: \\ Arellano, A. y Peralta, F. (2013): \\ "Autodeterminación de las personas \\ con discapacidad intelectual como \\ objetivo educativo y derecho básico: \\ estado de la cuestión", Revista \\ Española de Discapacidad, I (I): \\ 97-II7. \\ doi: <http://dx.doi.org/I0.5569/2340- \\ 5IO4.OI.OI.O5>
}

Fecha de recepción: 5-I I-2OI 2 Fecha de aceptación: 22-4-20I3 


\section{Introducción}

A lo largo de las últimas dos décadas, la autodeterminación ha generado gran interés en el campo de los estudios sobre las personas con discapacidad, dando lugar a un notable movimiento a favor del reconocimiento de este derecho de las personas con discapacidad intelectual y del desarrollo (Calkins et al., 20I I; Wehmeyer et al., 2000a, 2000b, 20II).

La autodeterminación es un concepto complejo y multidimensional; como constructo psicológico, se refiere al yo, a la acción causada y a la actuación volitiva de la persona (Walker et al., 20I I). De acuerdo con la teoría funcional de Wehmeyer (1998, I999, 2005, 2009), uno de los modelos conceptuales de mayor peso por su amplia difusión científica, se concibe la autodeterminación como un conjunto de actividades y habilidades necesarias para actuar como el agente causal o el protagonista de las propias acciones. En la actualidad, la promoción de la autodeterminación se considera un objetivo no sólo deseable, sino sobre todo posible y un indicador fundamental de la calidad de vida de estas personas (Calkins et al., 20I I; Vatlan et al., 20I I; Walker, et al., 20II; Wehmeyer et al., 20II). En este sentido, desde los años noventa, se ha venido sucediendo un creciente aumento de publicaciones, tanto en revistas científicas como en revistas más orientadas a la práctica, sobre teorías, modelos y programas de intervención relacionados con esta temática.

A lo largo de este artículo, se pretende aportar una visión sintética acerca del desarrollo actual de la autodeterminación como derecho básico de los jóvenes y adultos con discapacidad intelectual y otras discapacidades del desarrollo. Para ello, se presentan aquellas aportaciones teóricas y prácticas más destacadas, que constituyen una referencia de los esfuerzos realizados por la consecución de esta meta.

La metodología empleada en este trabajo ha consistido en seleccionar aquellas fuentes de la literatura que cumplieran al menos dos de estos tres criterios:

- Que estuvieran referidas específicamente a personas con discapacidad intelectual.

- Que se centraran en la autodeterminación como constructo o en alguno de sus elementos.

- Que aportaran información procedente de fuentes fiables.

Dichas fuentes consistieron básicamente las siguientes:

- Bases de datos académicas con algunas de las revistas más relevantes en materia de discapacidad y educación ${ }^{\mathrm{I}}$.

- Páginas webs de entidades dedicadas a la prestación de servicios para personas con discapacidad intelectual y sus familias, o bien a la investigación en este ámbito.

- Manuales de referencia publicados.

\section{Factores que impulsan el movimiento hacia la autodeterminación personal}

Aunque el concepto de autodeterminación tiene sus raíces históricas en la ciencia política y en la filosofía de los siglos XVII y XVIII, en el campo de la psicología aparece dentro de la psicología de la personalidad, ya en el siglo XX, con un candente debate acerca del determinismo y la libertad en la conducta humana, y se extiende al campo de la motivación cuando Deci y Ryan proponen una teoría de la motivación intrínseca, que lo incorpora como concepto central (Deci y Chandler, I986; Ryan y Deci, 200I).

\footnotetext{
I. Educational Resources Información Center (ERIC), Ingenta Connect o Google Scholar, ISI Web of Knowledge, Journal Citation Reports o Portal Latindex, a través de búsquedas con palabras clave, en español e inglés, como: discapacidad intelectual/retraso mental, autodeterminación, autonomía, autogestión, elección, toma de decisiones, empowerment. En dichas bases se encuentran algunas de las revistas con mayor índice de impacto a día de hoy.
} 
Cuando hablamos de la autodeterminación referida a personas con discapacidad intelectual, tomamos en consideración las dos acepciones básicas que tiene este concepto: a) como derecho humano básico y político (sentido sociopolítico); y b) como disposición personal a tener control sobre la propia conducta (Hughes y Agran, I998; Wehmeyer, I999, Walker et al., 20II; Wehmeyer et al., 2002). La importancia otorgada a este proceso como un derecho que hay que proteger y promover nos permite afirmar que la autodeterminación constituye ya el eje central en la planificación de los servicios dirigidos a las personas con discapacidad y a sus familias. Destacamos a continuación algunos de los factores que han generado el clima propicio para la emergencia de este movimiento, o bien han contribuido a su fortalecimiento.

\subsection{El desarrollo de nuevas legislaciones sobre los derechos civiles y la protección de las personas con discapacidad}

Desde que autores como Bank-Mikkelsen, Nirje o Wolfensberger enunciaran el principio de normalización, a comienzos de los años setenta, se han sucedido importantes avances legislativos conforme a los principios enunciados en la Declaración Universal de Derechos Humanos. Estas legislaciones pretenden ser garantes de los derechos civiles de las personas con discapacidad, y ofrecen un marco para el desarrollo de planes de acción orientados a capacitarlas (empowerment) y a hacerlas más autónomas (Wehmeyer y Ward, I995; Wehmeyer y Schwartz, I997) e iguales en dignidad y respeto (Wehmeyer et al., 20II). Algunas de ellas son, por ejemplo: en EE.UU., la Americans with Disabilies Act (ADA), de I990, la Individuals with Disabilities Education Act (IDEA), aprobada en I990 y ratificada en I997 y 2004, la Developmental Disabilities Act, de 2000; en Gran Bretaña, la All-Wales Mental Handicap Strategy, de I983; y, en España, la Ley I3/I982 de Integración Social de los Minusválidos (LISMI) o la Ley 39/2006 de Promoción de la Autonomía Personal y Atención a las Personas en Situación de Dependencia. De carácter más universal es la Convención
Internacional sobre los Derechos de las Personas con Discapacidad, aprobada por la Asamblea General de las Naciones Unidas en diciembre de 2006, con el compromiso de los Estados parte de promover, proteger y asegurar el goce pleno y en condiciones de igualdad de todos los derechos humanos y libertades fundamentales por todas las personas con discapacidad, y promover el respeto de su dignidad inherente.

A la luz de estas propuestas, consideramos que el creciente desarrollo de la autodeterminación en el campo de la discapacidad es una concreción del derecho universal al autogobierno y a la independencia de las personas y se ha convertido en un principio central de su lucha por la igualdad.

\subsection{La proliferación de movimientos organizados por personas con discapacidad y sus familias}

Desde mediados del siglo pasado, los padres de personas con discapacidad comienzan a agruparse para reclamar mejoras para sus hijos, transmitir sus necesidades de apoyo y exigir un mayor protagonismo en la toma de decisiones relativas a sus vidas (Turnbull et al., 2006). Especialmente importante es el proceso de desinstitucionalización que emprenden las familias en defensa de una mayor integración de sus hijos en la comunidad. Un poco más adelante, en los años setenta, nacen las primeras organizaciones de personas con discapacidad intelectual, cuyas acciones e iniciativas han ido cambiando la filosofía y configuración de los servicios, así como las expectativas sociales, y han favorecido una nueva conciencia social sobre sus capacidades y derechos, refrendada en las medidas legislativas a las que acabamos de referirnos. De estas organizaciones, nos interesa destacar las siguientes: el movimiento de vida independiente, los grupos de autoayuda, los grupos de autodefensa (por ejemplo, People First) o de autogestión. El mensaje principal de estas organizaciones, reflejado en el lema 'Nada sobre nosotros sin nosotros', ha propiciado el incremento de una identidad social positiva y su implicación activa en la planificación de las estrategias y políticas que les afectan. 
En España, y como botón de muestra, la Confederación Española de Organizaciones a favor de las Personas con Discapacidad Intelectual (FEAPS) ha venido desarrollando desde r 998 el Programa de Creación de Grupos de Autogestores. Recientemente, con motivo de la celebración del Año de la Ciudadanía, autogestores de todo el país pertenecientes al movimiento asociativo han elaborado un Manifiesto por la plena ciudadanía de las personas con discapacidad intelectual (2012) 2 . A través de esta declaración, las personas con discapacidad reclaman a la sociedad oportunidades para demostrar sus capacidades, información para tomar decisiones, entornos laborales, escolares y de ocio inclusivos, entre otras cuestiones. En definitiva, solicitan el compromiso de todos para ser reconocidos, verdaderamente, como ciudadanos de pleno derecho.

\subsection{El abandono del modelo del déficit}

Globalmente, durante las últimas décadas el modelo de discapacidad ha evolucionado desde un planteamiento centrado en los déficits hacia un enfoque de competencias y fortalezas, que reconoce que las personas con discapacidad también pueden asumir un mayor protagonismo en su vida. Buena muestra de ello son, por ejemplo, el modelo socioecológico de la discapacidad planteado por la Organización Mundial de la Salud (200I) o la reciente conceptualización de la discapacidad intelectual propuesta por la Asociación Americana sobre Discapacidad Intelectual y Discapacidades del Desarrollo (American Association on Intellectual and Developmental Disabilities, 20I0), que aborda la discapacidad como un constructo social y destaca el papel de los apoyos como medio para mejorar el funcionamiento humano.

Paralelamente, la emergencia de la llamada psicología positiva (Dykens, 2006; Linley et al.,

2. Dicho manifiesto fue leído el I9 de octubre de 20 I 2 en una jornada titulada 'Cádiz, cuna de los derechos', con la que la confederación quiso conmemorar el Bicentenario de la Constitución Española de I 8I 2.
2006; Seligman y Csikszentmihalyi, 2000) ha supuesto, sin duda, un importante apoyo a esta nueva conceptualización. Desde esta perspectiva se anima a examinar el potencial de las personas, más que a seguir centrando la atención en las deficiencias individuales e inmutables. En el campo de la educación especial, merece especial atención el trabajo de Elisabeth Dykens, investigadora, que realiza un llamamiento para que el mundo de la discapacidad participe de la psicología positiva:

Desde hace décadas nos hemos centrado en índices externos de calidad -condiciones físicas, conducta adaptativa...-, pero no sabemos nada sobre estados internos. ¿Qué mejor momento que ahora para preguntarnos qué necesitan las personas con discapacidad para ser felices, para tener una vida plena? (Dykens, 2006: I86).

\subsection{La apuesta por la calidad de vida}

También en los últimos años ha sido notable el progreso en la conceptualización del constructo calidad de vida de las personas con discapacidad y en el desarrollo de herramientas para medirla (Poston et al., 2003; Schalock, 2000; 2006; Schalock y Verdugo, 2010; Verdugo et al., 2009). Se concibe la calidad de vida como un constructo complejo que guía la práctica de los servicios de cara a mejorar las condiciones de vida de las personas que los reciben. Es, por tanto, un medio para alcanzar la equidad, la participación y una mayor satisfacción personal con la vida. Son ocho los dominios de calidad de vida internacionalmente aceptados: bienestar emocional, relaciones interpersonales, bienestar material, desarrollo personal, bienestar físico, autodeterminación, inclusión social y derechos (Schalock et al., 2002; 2005; 2010). En el marco de este modelo, algunas investigaciones (como las de Nota et al., 2007; o Walker et al., 20II) han probado que la autodeterminación juega un papel mediador en el incremento de la calidad de vida. 


\subsection{Los avances en la filosofía y metodología de los servicios}

Coincidiendo con los cambios en la consideración de la discapacidad y con el reconocimiento de sus derechos, incluido su derecho a la autodeterminación, también las organizaciones han ido desarrollando una nueva visión de sus servicios. Así, han tomado como centro a la persona, con la pretensión de llegar a conocer lo que es deseable para ella y hacerlo posible; es decir, 'hacer lo deseable posible'. La persona, y su entorno, es el foco de la planificación, poniéndose un mayor énfasis en la toma de decisiones de los usuarios o clientes de los diferentes servicios y, por tanto, en la necesidad de consultarles. Esta serie de cambios no sólo suponen un nuevo modo de entender el fenómeno de la discapacidad, sino que también conllevan modificaciones sustanciales en las prácticas profesionales. De este modo, se adoptan nuevos enfoques que tienen como premisa elemental la idea de que el control debe trasladarse de quienes proporcionan los servicios hacia quienes los reciben (Wehmeyer et al., 2000a, 200ob).

Basándose en principios como la inclusión o el empowerment, los profesionales desarrollan metodologías de intervención que permiten llevar a la práctica estos planteamientos. Especialmente importantes en la promoción de la autodeterminación son, por ejemplo, la planificación centrada en la persona (Arteta et al., 201 2; Brown et al., 2007; Heller et al., 20II) o el enfoque centrado en la familia (Leal, I999; Peralta y Arellano, 20I0).

Además de los factores recién citados, se han sucedido de forma simultánea avances en la investigación que aportan evidencias acerca de este concepto y de su relación con objetivos como la vida independiente, el acceso al empleo, etc. Son muchas las iniciativas y proyectos que, desde una perspectiva científica, ahondan en el qué y el cómo de la autodeterminación de las personas con discapacidad. Destacamos, por ejemplo, la Conferencia Nacional sobre Autodeterminación, organizada en 1989 por el National Institute for Disability and
Rehabilitative Research; el Programa para la Autodeterminación de Personas con Discapacidades del Desarrollo", puesto en marcha enI 996 y financiado por la Fundación Robert Wood Johnson (Ward y Kohler,I996); el New Hampshire Self-Determination Project, iniciado en I 993 por Monadnock Developmental Services of Keene (Wehmeyer y Stancliffe, 2003); la National Training Initiative, en 2008 , a cargo de investigadores de cinco universidades en EE.UU. lideradas por la Universidad de Missouri; o las variadas acciones de la European Agency for Development in Special Needs Education, una organización independiente creada por los Estados miembros de la Unión Europea para promover la inclusión de las personas con necesidades educativas especiales. En el caso de España, cabe señalar los proyectos de investigación llevados a cabo por el Instituto Universitario de Integración en la Comunidad (INICO), formado por profesionales de la Universidad de Salamanca, y de otras universidades. Algunos autores han revisado las evidencias generadas por la investigación, que pueden dar una idea sobre el estado de la cuestión en autodeterminación (por ejemplo, Algozzine et al., 200I; Chambers et al., 2007; Cobb et al., 2009; Karvonen et al., 2004; Calkins et al., 20I I; o Wood et al., 2005).

3. Autodeterminación: su conceptualización actual

De acuerdo con lo expuesto, los esfuerzos a favor de la autodeterminación han partido tanto del contexto político como del socioeducativo o científico, y se aplican a todas las áreas significativas de la vida de las personas con discapacidad y de sus familias.

En los últimos veinte años, como venimos insistiendo, la conceptualización o fundamentación teórica del constructo de autodeterminación ha suscitado una amplia discusión. En la actualidad, se aprecia un alto grado de acuerdo entre los expertos a la hora 
de considerar que son tres los marcos teóricos en los que se fundamenta la comprensión del constructo (Walker et al., $20 \mathrm{II}$; Wehmeyer et al., 20I I $)^{3}$. Dichos modelos, cuyas características se sintetizan en el Cuadro I, han sido desarrollados y validados en el campo de la discapacidad y comparten las siguientes características (para más información, se puede consultar el trabajo de Wehmeyer et al., 2003):

- El desarrollo de la autodeterminación es un proceso que dura toda la vida, aunque cobra especial importancia en determinadas etapas (fundamentalmente, durante la transición a la vida adulta) [Wehmeyer y Field, 2007].

- Los atributos internos y la oportunidad del contexto influyen en el grado de autodeterminación de la persona, que aprende a ser responsable y principal protagonista de sus acciones a través de la interacción con su entorno (Lee et al., 20I2; Wehmeyer et al., 2009).

- Todas las personas tienen potencial para desarrollar su autodeterminación, incluso aquellas con discapacidades más severas (Chambers et al., 2007; Wehmeyer et al., 2OII).

- La familia es uno de los contextos de mayor influencia en la adquisición y ejercicio de las habilidades de autodeterminación. En dicho contexto, todos los miembros ejercen como apoyos y modelos de conducta para las personas con discapacidad (Arellano, 20I 2; Peralta, 2008; 20I I; Turnbull y Turnbull, I996, 2000, 200I).

De los modelos citados, por su gran difusión en nuestro entorno y su fecunda investigación, el modelo funcional de autodeterminación de M. L. Wehmeyer merece una descripción más detallada, aunque también necesariamente sintética, para no exceder la extensión de este trabajo.

3. Además de los tres modelos citados, es preciso también reconocer el modelo desarrollado por Field et al. (I998a, I999b), por su contribución a la clarificación del término autodeterminación.

\subsection{Modelo funcional de autodeterminación}

Wehmeyer define la conducta autodeterminada como aquella acción volitiva que lleva al sujeto a actuar como el principal agente causal en las cuestiones relevantes de su vida, sin influencias externas innecesarias, y que le permite mantener y mejorar su calidad de vida (Wehmeyer, I998, I 999, 2004, 2005, 2009). Las conductas, por tanto, son autodeterminadas en la medida en que sirven a la persona para obtener un fin o generar un cambio. Son cuatro las características esenciales de estas conductas:

- Autonomía. La persona actúa de acuerdo a sus propias preferencias, intereses o capacidades y de manera independiente, en un proceso de individuación que lleva de la dependencia a la interdependencia.

- Autorregulación. La persona examina su ambiente y sus repertorios de respuesta para tomar decisiones sobre cómo y cuándo actuar, así como para evaluar y revisar, cuando sea necesario, sus planes de acción en función de los resultados. La clave está en el ajuste, es decir, en el examen de la eficacia de la estrategia empleada, y en el cambio -si es necesario- de la estrategia o conducta (Wehmeyer y Field, 2007).

- Creencias de control y eficacia. La persona inicia y responde a los acontecimientos con dominio cognitivo (eficacia personal), dominio personal (locus de control) y dominio motivacional. El grado de control depende de las creencias que las personas tienen sobre su capacidad para llevar a cabo las conductas necesarias dirigidas a obtener unos resultados y para lograr dichos resultados si realizan tales conductas.

- Autoconciencia y autoconocimiento. La persona actúa con un conocimiento comprensivo y razonablemente preciso de sí misma y de sus puntos fuertes y limitaciones, y emplea este conocimiento para alcanzar metas personalmente valiosas.

La modalidad de expresión de estas características varía con la edad, la oportunidad, 
Cuadro 1. Síntesis de los modelos teóricos de autodeterminación más relevantes

\begin{tabular}{|c|c|c|c|}
\hline & $\begin{array}{l}\text { Modelo funcional de } \\
\text { M. Wehmeyer (1999, } \\
2000,2004,2009)\end{array}$ & $\begin{array}{l}\text { Modelo ecológico } \\
\text { tripartito de B. Abery y R. } \\
\text { Stancliffe (2003a, 2003b) }\end{array}$ & $\begin{array}{l}\text { Modelo de } \\
\text { autodeterminación como } \\
\text { autorregulación de D. } \\
\text { Mithaug (Mithaug, 2003; } \\
\text { Mithaug et al., 2003) }\end{array}$ \\
\hline $\begin{array}{l}\text { ¿Qué entienden por } \\
\text { autodeterminación? }\end{array}$ & $\begin{array}{l}\text { Aquellas acciones } \\
\text { volitivas que capacitan } \\
\text { a la persona para } \\
\text { actuar como el agente } \\
\text { causal primario de su } \\
\text { propia conducta y para } \\
\text { mantener o mejorar su } \\
\text { calidad de vida. }\end{array}$ & $\begin{array}{l}\text { Proceso complejo que tiene } \\
\text { como meta última lograr } \\
\text { el control personal que el } \\
\text { sujeto desea en aquellas } \\
\text { áreas de su vida que } \\
\text { percibe como importantes. }\end{array}$ & $\begin{array}{l}\text { El resultado de la interacción } \\
\text { entre la capacidad de la } \\
\text { persona y la oportunidad } \\
\text { social. }\end{array}$ \\
\hline $\begin{array}{l}\text { ¿Cuándo decimos } \\
\text { que una persona es } \\
\text { autodeterminada? }\end{array}$ & $\begin{array}{l}\text { Cuando actúa de } \\
\text { modo autónomo y } \\
\text { autorregulado, inicia } \\
\text { acciones y responde a } \\
\text { los acontecimientos con } \\
\text { un sentido de control y } \\
\text { competencia personal, } \\
\text { y actúa según un } \\
\text { conocimiento razonable } \\
\text { de sí mismo. }\end{array}$ & $\begin{array}{l}\text { Cuando ejerce control sobre } \\
\text { aquellas áreas importantes } \\
\text { de su vida o cede } \\
\text { voluntariamente el control } \\
\text { de ciertas decisiones a } \\
\text { personas de confianza. }\end{array}$ & $\begin{array}{l}\text { Cuando la persona libre } \\
\text { de influencias externas, } \\
\text { autorregula sus elecciones } \\
\text { y acciones para obtener } \\
\text { la meta deseada. Siempre } \\
\text { ocurre en un contexto social. }\end{array}$ \\
\hline $\begin{array}{l}\text { ¿Cuáles son los } \\
\text { componentes } \\
\text { de una conducta } \\
\text { autodeterminada? }\end{array}$ & $\begin{array}{l}\text { - } \text { Autonomía. } \\
\text { - } \text { Autorregulación. } \\
\text { - y eficacia. } \\
\text { - Autoconciencia y } \\
\\
\text { autoconocimiento. }\end{array}$ & $\begin{array}{l}\text { - Grado de control } \\
\text { ejercido. } \\
\text { - Grado de control } \\
\text { deseado. } \\
\text { - Importancia atribuida } \\
\text { a las áreas en las que } \\
\text { ejercer el control. }\end{array}$ & $\begin{array}{l}\text { - Autoconocimiento y } \\
\text { autoconciencia. } \\
\text { - Capacidad de } \\
\text { autorregulación. } \\
\text { - Recursos personales, } \\
\text { sociales y económicos. }\end{array}$ \\
\hline
\end{tabular}

Fuente: Elaboración propia.

la capacidad y el contexto (Wehmeyer et al., 2OII); asimismo, emergen en cuanto la persona desarrolla y adquiere elementos o componentes de la conducta autodeterminada, como hacer elecciones, tomar decisiones, resolver problemas, establecer metas y logros, expresar habilidades de autorregulación y autodirección, adquirir habilidades de autodefensa y liderazgo, desarrollar percepciones positivas de control y eficacia y expectativas de resultados, tener autoconciencia o autoconocimiento. Todo ello da lugar a manifestaciones de la conducta autodeterminada en grados y niveles diferentes, que deben ser evaluados mediante distintos procedimientos (como se refleja en el siguiente apartado), de cara a proponer planes de intervención acordes con las necesidades actuales de la persona.

\subsection{Proceso de evaluación de la} autodeterminación: instrumentos de medida y aspectos metodológicos

La valoración de las conductas autodeterminadas de la persona con discapacidad permite identificar sus necesidades o bien determinar la efectividad de la instrucción. Esta tarea requiere adoptar la perspectiva de la empowerment evaluation, tratando de llevar a la persona (normalmente en una situación de dependencia y de escaso control) hacia la reflexión, la autoayuda, la autoevaluación y el sentido de autodeterminación (Fetterman, I996). La situación de evaluación se transforma así en un proceso más colaborativo que prescriptivo, al permitir la identificación por parte de la persona 
de sus puntos fuertes y débiles y, por tanto, constituye una oportunidad para la mejora.

Entre las técnicas empleadas como fuentes de información para la toma de decisiones, queremos destacar las siguientes.

- Observación sistemática: es uno de los mejores medios para evaluar la competencia en un entorno natural, así como las condiciones de los contextos en que se desenvuelve la persona (por ejemplo, el contexto familiar).

- Entrevista semiestructurada con cada uno de los agentes implicados: persona con discapacidad, profesionales y familias. Especialmente compleja es la entrevista a personas con discapacidad intelectual, por la necesidad de adaptar el proceso para obtener información fiable. Algunas recomendaciones, como las que ofrecen Finlay y Lyons (200I) o Malik et al. (I99I), que se resumen al final de este epígrafe, pueden guiar al evaluador en esta tarea.

- Informe evolutivo del alumno. En entornos formales, cuando la evaluación la lleva a cabo el educador en vistas a introducir cambios curriculares, es uno de los primeros recursos. Además, pueden resultar útiles herramientas como el portafolios o la planificación centrada en la persona, por la gran cantidad de información que reúnen sobre la persona (preferencias, competencias, intereses, relaciones, entre otras).

Finalmente, están los instrumentos estandarizados destinados a medir globalmente el grado de autodeterminación o alguna de sus dimensiones. De ellos, por su reconocimiento internacional, presentamos estos cuatro:

a. Escala de autodeterminación ARC

(Wehmeyer y Kelchener, I995)

Consta de 72 ítems, administrados en

forma de autoinforme a los adolescentes y adultos con discapacidad intelectual, y agrupados en cuatro secciones: autonomía, autorregulación, creencias de control y eficacia, y autoconciencia. Se obtienen cinco puntuaciones, una por cada sección, y una puntación global de autodeterminación. Esta escala ha sido traducida y adaptada al español tanto en su versión para adolescentes como en su versión para adultos. La primera versión, a cargo de Wehmeyer et al. (2006), se aplica en formato de entrevista semiestructurada y ha obtenido unos índices de fiabilidad similares a los de la escala original (alpha de Cronbach 0,90 en la versión para adolescentes y o,8o en la de adultos). Una segunda adaptación está todavía en proceso de validación (GómezVela et al., 2012; Verdugo, 2006).

b. Escalas de Minnesota (Abery et al., 2000) Las escalas de Minnesota se estructuran en cinco subescalas:

- Escala de ejercicio del control (grado de control que la persona ejerce en actividades de diferentes áreas de su vida).

- Escala de preferencia en la toma de decisiones (grado de control deseado, independientemente del que realmente ejerce).

- Escala de importancia del control en diferentes aspectos de la vida.

- Escala de conocimientos, actitudes y destrezas de autodeterminación (contiene dos formatos: uno para la persona con discapacidad y otro para los profesionales que trabajan con ella).

- Escala sobre el entorno (que sólo cumplimentan los profesionales).

Este instrumento tiene la ventaja de contar con pictogramas que facilitan la comprensión de sus ítems a las personas con limitaciones psicolingüísticas.

c. Batería de evaluación de la autodeterminación (Hoffman et al., 2004a, 2004b)

Consta de cinco instrumentos 4

4. Disponibles en la página del Zarrow Center for Learning Enrichment, de la Universidad de Oklahoma: <https://education.ou.edu/zarrow/? $\mathrm{p}=38 \& \mathrm{z}=4 \mathrm{I}>$ (consultada el $29 \mathrm{de}$ octubre de 20I2). 
- Escala de conocimientos sobre autodeterminación.

- Escala de percepción de la autodeterminación para padres.

- Escala de percepción de la autodeterminación para profesores.

- Listado de observación de la autodeterminación.

- Escala de autodeterminación para el alumno.

Esta batería de pruebas resulta bastante completa y se presta a múltiples usos. Por una parte, permite identificar las discrepancias entre la visión de profesionales, padres y persona con discapacidad, aspecto especialmente interesante. Por otra, aporta una visión integral, dado que se centra en variables de tipo cognitivo (conocimientos, creencias), afectivo (sentimientos) y conductual (comportamientos autodeterminados).

d. Escala de autodeterminación AIR (Wolman et al., I994)

Permite recabar información acerca de la autodeterminación como constructo global. Está basada en la teoría de aprendizaje autodeterminado de Dennis Mithaug (Mithaug et al., I998). Consta de una forma para padres, otra para alumnos y otra para profesores $^{5}$. Evalúa dos amplios componentes de la autodeterminación: por una parte, las capacidades del alumno y, por otra, las oportunidades que le brindan los diferentes contextos (centro educativo y entorno familiar). Esta escala permite elaborar un perfil acerca del nivel de autodeterminación del alumno, en el que aparecen tanto sus puntos fuertes como sus áreas de mejora. Dicho perfil sirve para identificar metas individuales significativas que incorporar en los planes educativos.

Las técnicas e instrumentos descritos de forma necesariamente concisa son una ayuda importante en el proceso de evaluación, ya que

5. También disponibles en el Zarrow Center for Learning Enrichment: <http://www.ou.edu/zarrow/sdetermination.htm> (consultada el 29 de octubre de 20I2). proporcionan un estándar con el que comparar qué características deben ser medidas, pero este proceso, como ya se ha señalado, no está exento de dificultades metodológicas. Algunas de las más frecuentes se enumeran a continuación

(Dykens, 2006; Finlay y Lyons, 200I):

- Contenido de los ítems. Las personas con discapacidad pueden presentar una limitada competencia para su autoevaluación (Wehmeyer et al., I998) y para comprender algunos conceptos que requieren pensamiento abstracto, perspicacia o cierto dominio del lenguaje (Dykens, 2006). Por ello, serán necesarias adaptaciones que eviten las dificultades cognitivas y lingüísticas (vocabulario claro, confirmar la comprensión de las preguntas, incluir preguntas sobre acontecimientos de la vida de la persona, entre otras).

- Enunciado de los items con estructuras lingüisticas complejas. Los ítems formulados en forma pasiva, con marcadores temporales complejos o con partículas negativas, pueden generar confusión a la hora de dar la respuesta.

- Normas de aplicación. El protocolo de aplicación ha de ser más flexible, colaborativo y participativo. A menudo, las preguntas deberán ser reformuladas, adaptadas o simplificadas y se hacen imprescindibles los ejemplos, las repeticiones o el empleo de varios turnos de pregunta-respuesta para cada ítem, así como el uso de otro tipo de apoyos visuales, como los pictogramas.

- Interpretación de los resultados. Una puntuación baja puede reflejar escasez de oportunidades, capacidades limitadas de la persona o ambas cosas. La puntuación en los tres casos puede ser la misma, pero la toma de decisiones respecto a la evaluación, no (Wehmeyer, I995). Es imprescindible, por tanto, escuchar atentamente sus respuestas, hacer las preguntas de manera correcta, prestarle los apoyos que necesite para que pueda responder, contrastar sus respuestas con otras fuentes de información y controlar su mayor tendencia a la aquiescencia (Finlay y Lyons, I998). 
Una evaluación realizada con las suficientes garantías metodológicas permitirá obtener la información válida para incrementar las oportunidades dirigidas a capacitar a las personas con discapacidad intelectual. De este modo, podrán hacer frente con éxito a las complejidades de la vida adulta, ya que se basarán en la valoración de sus necesidades, intereses y preferencias.

4. Propuestas educativas: la autodeterminación una meta posible

El logro de la autodeterminación personal es una tarea compleja, pero, como se ha querido reflejar en este artículo, posible. El ejercicio de este derecho por parte de las personas con discapacidad depende, en parte, de la existencia de comunidades de aprendizaje que posibiliten su participación activa. En este camino hacia la autodeterminación, no podemos olvidar el importante papel de cada uno de los agentes educativos presentes en la vida de la persona con discapacidad. Diversos estudios, de hecho, ponen de manifiesto cómo las percepciones, creencias y actitudes de los profesionales de los servicios y de los padres comprometen la adquisición y ejercicio de habilidades de este tipo (Arellano, 201 2; Doss y Hatcher, I996; Peralta, 2008; Peralta et al., 2005; Wehmeyer, 2005; Zulueta y Peralta, 2008). Por lo tanto, educar en autodeterminación conlleva, con frecuencia, un profundo cambio en la manera de concebir y planificar la provisión de apoyos en el contexto escolar, social y familiar (Field y Hoffman, 2002; Whemeyer y Field, 2007). Y, tal y como expone Nisbet (2000), involucrar a toda la comunidad educativa para lograr una verdadera transformación supone, en primer lugar, definir claramente el rumbo del cambio (el qué) y, en segundo lugar, desarrollar una metodología para lograrlo (el cómo). Las siguientes propuestas de intervención pueden ser de ayuda a la hora de iniciarnos en este segundo paso.

\subsection{Intervención en el ámbito educativo formal}

Tradicionalmente, el modelo de enseñanza adoptado con alumnos considerados con necesidades especiales ha estado centrado en el profesional, ha sido altamente estructurado, incluso rígido, y ha dejado poco lugar a la participación activa del alumno. El desarrollo de la conducta autodeterminada exige cierto traspaso de responsabilidad hacia la persona con discapacidad que le permita, poco a poco, llevar las riendas de su propio proceso educativo. En ese sentido, cualquier iniciativa dirigida a este fin debe tener en cuenta los siguientes principios:

- Tomar como base un modelo de enseñanza inclusiva que propicie la integración y participación en la comunidad.

- Adoptar una perspectiva de capacitación (empowerment) de la persona, en consonancia con los principios de dignidad individual, responsabilidad y orientación para la carrera.

- Asumir un estilo de enseñanza no directivo y centrado en el alumno, creando contextos de aprendizaje interactivos y cooperativos.

Además de este cambio de actitud, diversos estudios muestran la eficacia de determinados currículos y modelos de enseñanza para promover la autodeterminación. Dados los resultados positivos encontrados, actualmente son innumerables las propuestas centradas en este objetivo. Presentaremos en estas páginas algunos de los programas más significativos ${ }^{6}$ :

a. Modelo de Instrucción para el Aprendizaje Autodeterminado (Self-determined Learning Model of Instruction, SDLMI) [Mithaug et al., 1998]

Este modelo se centra en el desarrollo y adquisición de habilidades de autorregulación, en el ámbito de enseñanzaaprendizaje. Este tipo de instrucción tiene un impacto positivo en la autodeterminación; los estudiantes autodeterminados son estudiantes autorregulados (Agran et al.,

6. Para una revisión más detallada sobre estos y otros materiales, se puede consultar la publicación de Peralta et al. (2006). 
2008). Básicamente, el SDLMI plantea un proceso de resolución de problemas con el que los alumnos aprenden en diversas áreas y actividades curriculares a establecer una meta (fase I), a desarrollar un plan de acción para lograr dicha meta (fase 2), y a modificar su meta o su plan de acción, en función de los resultados (fase 3 ). En cada una de estas fases, el alumno tiene que responder a cuatro preguntas: ¿qué quiero conseguir?, ¿cuáles son las posibles soluciones a mi problema?, ¿qué obstáculos puedo encontrarme? y ¿qué consecuencias conlleva cada opción? Se dirige a alumnos con y sin discapacidad de todas las edades.

Además, tomando como referencia este modelo de aprendizaje autorregulado, se ha desarrollado una guía para padres y niños más pequeños (A Parent's Guide to the Self-determined Learning Model for Early Elementary Students [Palmer y Wehmeyer, 2002]), un programa dirigido a alumnos con discapacidad de entre I4 y 2 I años (Whose Future is it Anyway?: [Wehmeyer et al., 2004]) y un modelo de instrucción para alumnos de entre I 8 y 2 I años (Beyond High School [Wehmeyer et al., 2008]).

b. Take Charge [Powers et al., I996] Basado en las teorías de la motivación de logro y expectativas de autoeficacia, Take Charge tiene como objetivo reducir el riesgo de indefensión aprendida al que están expuestos alumnos con necesidades especiales. Está dirigido a alumnos de entre I 3 y I 6 años con discapacidad física, intelectual, dificultades de aprendizaje o trastornos de tipo emocional. También puede ser utilizado en grupos de alumnos sin discapacidad. El papel del profesional es el de un facilitador, dado que éste es un programa centrado, fundamentalmente, en los alumnos. Los cuatro elementos sobre los que se fundamenta esta propuesta son los siguientes:

- El desarrollo de capacidades del alumno. Implica ofrecer oportunidades para la toma de decisiones, la solución de problemas, el establecimiento de metas, el acceso a información sobre los recursos disponibles y la disposición de los apoyos necesarios.

- El mentorazgo. Consiste en establecer una relación con una persona, de características e intereses similares, que actúe de modelo y que se comprometa a guiar al alumno y a apoyarle en el desarrollo de sus metas.

- El apoyo de los iguales. A través de la participación en talleres (sobre temas acerca de la discapacidad, la vida adulta) y mediante el establecimiento de relaciones significativas entre los participantes.

- El apoyo de los padres. A lo largo de todo el proceso se promueve activamente la colaboración entre jóvenes, padres y profesionales para aumentar las oportunidades de ejercer la autodeterminación en el ambiente familiar. Con el objetivo de responder a las necesidades de cada familia, se les proporciona información, materiales escritos, se les presta ayuda técnica y se les ofrecen cursos formativos.

c. Un currículo hacia la autodeterminación: pasos (Steps to Self-determination. A Curriculum to Help Adolescents Learn to Achieve Their Goals) [Field y Hoffman,r996; y Hoffman y Field, 2006]

Se estructura en torno a cinco componentes: a) conócete a ti mismo y a tu entorno; b) valórate; c) planifica; d) actúa; y e) aprende. Tiene como objetivo fundamental ayudar a los alumnos en etapa de transición a la vida adulta a definir y lograr aquellas metas que son importantes para ellos. Está dirigido a alumnos de secundaria con y sin discapacidad, y se puede impartir de formas muy variadas: como una actividad extraescolar, un curso específico o una actividad integrada en el currículum. Se desarrolla a través de I 7 sesiones de unos 55 minutos, en las que participan alumnos y profesor. Este último actúa como copartícipe del proceso de aprendizaje y debe encargarse de proporcionar modelos adecuados y de crear un clima de clase colaborativo. Por otra 
parte, se incluyen también actividades en las que puedan participar la familia $u$ otras personas significativas que apoyen al alumno.

El paquete completo de este currículum incluye una guía para el facilitador, materiales audiovisuales, libro de actividades para el alumno y el instrumento anteriormente señalado para evaluar autodeterminación: Self-determination Knowledge Scale (Hoffman et al., 2004a, 2004b).

d. Programa de autodeterminación ChoiceMaker (Martin y Marshall, I996, I997)

El programa de autodeterminación ChoiceMaker tiene como objetivo que el alumno elabore y dirija su propia planificación educativa, mediante el establecimiento de metas personales durante la etapa de transición a la vida adulta. Pretende, por lo tanto, fomentar la responsabilidad del alumno y capacitarle para usar estrategias de autogestión, autoevaluación o de reajuste de metas, entre otras.

Se estructura en torno a tres secciones generales, que plantean contenidos y objetivos diversos, a lo largo de varias áreas de la vida de la persona con discapacidad. Se desarrollan un conjunto de módulos, con sus correspondientes lecciones, en cada una de las dimensiones y áreas. Cada uno de los módulos se acompaña de vídeos y materiales para el alumno.

Centrándonos en nuestro país, encontramos en FEAPS una fuente de recursos prácticos con multitud de estrategias que llevar a cabo en los entornos formales. Recogemos en el Cuadro 3 algunos materiales que pueden servir de referencia y que se encuentran disponibles en la página de la entidad, para su descarga gratuita.

Es preciso añadir que estos materiales están basados en modelos de relevancia internacional ya citados (como, por ejemplo, el modelo funcional de Michael Wehmeyer) y que, por tanto, comparten los principios de la cultura de autodeterminación que se ha descrito en estas páginas.

\subsection{Intervención en el ámbito familiar}

Si bien los profesionales que trabajan en el ámbito de la discapacidad están más familiarizados con la autodeterminación, al menos en su sentido fundamental, en el caso de las familias, esto es menos frecuente, aunque muchas de ellas reconocen que uno de sus objetivos principales en cuanto a su hijo con

Cuadro 2. Estructura y objetivos de ChoiceMaker

\begin{tabular}{|c|c|c|c|}
\hline Niveles/áreas & Eligiendo metas & Expresando metas & $\begin{array}{l}\text { Llevando a cabo las } \\
\text { acciones }\end{array}$ \\
\hline $\begin{array}{l}\text { Educación } \\
\text { secundaria }\end{array}$ & \multirow{6}{*}{$\begin{array}{l}\text { Enseña al alumno las } \\
\text { habilidades necesarias } \\
\text { para identificar sus } \\
\text { intereses y metas en } \\
\text { una o varias áreas de } \\
\text { transición. }\end{array}$} & \multirow{6}{*}{$\begin{array}{l}\text { Centrada en la adquisición } \\
\text { de habilidades de liderazgo } \\
\text { para gestionar su propio plan } \\
\text { educativo, comunicar sus } \\
\text { intereses y preferencias, o } \\
\text { dirigir reuniones. }\end{array}$} & \multirow{6}{*}{$\begin{array}{l}\text { Ayuda al alumno a definir } \\
\text { cómo va a lograr su meta: } \\
\text { qué pasos tiene que dar, } \\
\text { cuáles son los objetivos } \\
\text { específicos, qué apoyos } \\
\text { necesita, cómo va a evaluar } \\
\text { el progreso, entre otras } \\
\text { cuestiones. }\end{array}$} \\
\hline Empleo & & & \\
\hline $\begin{array}{l}\text { Educación } \\
\text { postescolar }\end{array}$ & & & \\
\hline $\begin{array}{l}\text { Asuntos } \\
\text { personales }\end{array}$ & & & \\
\hline $\begin{array}{l}\text { Vivienda y vida } \\
\text { diaria }\end{array}$ & & & \\
\hline $\begin{array}{l}\text { Participación en la } \\
\text { comunidad }\end{array}$ & & & \\
\hline
\end{tabular}

Fuente: Elaboración propia a partir de Martin y Marshall (I996, I997). 
La meta de esta guía es ayudar a las personas con discapacidad intelectual en tres tareas:

- Conocer cuáles son sus derechos, utilizando la Convención de Naciones Unidas de los Derechos de las Personas con Discapacidad.

- Evaluar si se están respetando estos derechos en su día a día.

- Poner en marcha algunas acciones para defender y reivindicar sus derechos.

Autodeterminación. Guía para personal de apoyo (2009)

Unidades que se trabajan: 1) 'Hacer elecciones'; 2) 'Tomar decisiones'; 3) 'Plantearnos objetivos';

4) 'Resolver problemas'; 5) 'Conocernos mejor'; 6) 'Defendernos'; 7) 'Ser más independientes'.

La guía viene acompañada por material en formato audiovisual contenido en un cedé y veinte fichas para trabajar con las personas con discapacidad.

La autodeterminación de las personas con discapacidad intelectual (2002)

Además de un marco teórico, se presenta un compendio de buenas -y malas- prácticas en ámbitos como vida en el hogar, ocio y tiempo libre, formación y ocupación y vida en la comunidad.

Fuente: elaboración propia a partir de las publicaciones de FEAPS (<http://www.feaps.org/>).

discapacidad es desarrollar su autonomía lo más posible. Este conocimiento más básico de lo que implica la autodeterminación por parte de las familias plantea la necesidad de ofrecerles una mayor formación al respecto (Arellano, 2012; Chambers et al., 2007; Erwin et al., 2009; Zulueta y Peralta, 2008). Cuando se les ayuda a adquirir competencias y se les proporcionan apoyos, son capaces de mejorar y apoyar las habilidades de autodeterminación de sus hijos (Abery et al., I995).

El entorno del hogar cumple sus funciones, por una parte, como un lugar físico y, por otra, como un ámbito de cuidado y de satisfacción de las necesidades psicosociales. Atendiendo a este doble papel, y centrándose sobre todo en la infancia, son varias las sugerencias que autores como Abery y Zajac (I996), Brotherson et al., (2008), Cook et al., (2007), Doss y Hatcher (1996), Erwin et al., (2009), Illán et al. (2005) o Matuszewski (r996) plantean.

En primer lugar, los padres deben favorecer la interacción del niño con discapacidad con su entorno, físico y social (por ejemplo, permitiendo que tenga acceso a sus objetos personales, pudiendo ordenarlos y usarlos de manera autónoma; o procurando que el niño pueda estar, jugar e interaccionar con el resto de miembros de la familia). En segundo lugar, cualquier actividad de la vida diaria permite practicar la toma de decisiones. Las familias, a través de pequeñas acciones, pueden contribuir a que la persona con discapacidad funcione de modo autónomo o, en la medida de lo posible, participe de elecciones diarias: qué tipo de actividad realizar, con qué materiales, con qué personas, dónde, cuándo, decidir no participar, decidir cuándo acabar, por ejemplo. En tercer lugar, los niños, desde muy pequeños, controlan y regulan el entorno en que se mueven de modos diversos. En el caso de aquellos con discapacidad, los padres juegan un rol más activo e intencional a la hora de proporcionarles oportunidades para ello. Por último, el entorno familiar, por la seguridad y el afecto incondicional que ofrece, es un medio privilegiado para la adquisición de una autoestima adecuada. A este respecto, quizá el aspecto más complicado para las familias reside en cómo abordar el conocimiento sobre la propia discapacidad y las limitaciones que de ella se derivan. A pesar de esta dificultad, es posible hablar acerca de la discapacidad en la familia con naturalidad, sin dejar que ésta sea el único rasgo que defina a la persona.

Guardando muchas similitudes con todas estas estrategias, Davis y Wehmeyer publican en I99I diez pasos hacia la independencia, dirigidos a padres y madres, que proporcionan un marco 
útil para cualquier propuesta de formación para familias (véase Cuadro 4).

Además de estrategias generales como éstas, encontramos tres herramientas que son ejemplos significativos del trabajo que durante los últimos años se ha llevado a cabo en torno a esta área: la guía para padres basada en el modelo de aprendizaje autorregulado de Palmer y Wehmeyer (2002); el currículum familiar de Abery et al., (I994); y el manual de formación para familias en el apoyo a la autodeterminación de Ponce (2010), editado por FEAPS. Recogemos en el Cuadro 5 los principales rasgos de estos recursos.

En vista de todo lo dicho, consideramos que la persona con discapacidad, cuando cuenta con oportunidades suficientes, es capaz de ganar, paso a paso, autodeterminación. Las propuestas de intervención aquí recogidas esperamos que aporten a profesionales y familias orientaciones útiles que les ayuden a ejercer con éxito sus roles como apoyos y facilitadores de autodeterminación.

\section{Conclusiones y perspectivas de futuro}

Actualmente, la autodeterminación es un pilar básico sobre el que sustentar la planificación de los servicios y programas destinados a mejorar la calidad de vida de las personas con

\section{Cuadro 4. Pasos hacia la autodeterminación de Davis y Wehmeyer (1991)}

1. Lograr un equilibrio entre la protección necesaria y la independencia, permitiendo a sus hijos explorar su propio mundo.

2. Hacer que sus hijos entiendan que lo que dicen y lo que hacen es importante, y que pueden influir en los demás. Animarles a que asuman riesgos, a que hagan preguntas y a que muestren sus opiniones.

3. Cultivar un sentido de autoestima positivo. Los padres tienen que transmitir a sus hijos la idea de que son importantes para los demás y de que merece la pena emplear el tiempo con ellos.

4. No rehuir las preguntas de su hijo relacionadas con su discapacidad. Destacar el hecho de que cada persona es única, resaltar las capacidades y apoyar la aceptación de aquellas limitaciones inevitables.

5. Valorar los procesos orientados a metas y no sólo los resultados o el rendimiento. Favorecer la adquisición de habilidades relacionadas con la organización y el establecimiento de objetivos, modelando dichas conductas.

6. Favorecer la interacción con otros niños y personas de edades y capacidades diferentes (con y sin discapacidad, dentro y fuera del entorno del hogar).

7. Establecer expectativas realistas pero ambiciosas. Celebrar las experiencias de éxito, a la vez que se protege ante el fracaso continuado en tareas difíciles o imposibles de conseguir.

8. Proporcionar oportunidades para que asuman la reponsabilidad de sus propias acciones, éxitos o fracasos.

9. No dejar al azar las oportunidades para realizar elecciones. Asegurarse de que la persona con discapacidad elija en actividades y situaciones significativas para ella y de que se respete su decisión.

10. Proporcionar feedback positivo y honesto, transmitiendo la idea de que todas las personas, incluidos los padres, cometen errores. Hacerles comprender que siempre es posible buscar tareas, objetivos y metas alternativas asequibles a las posiblidades de uno mismo.

Fuente: Elaboración propia a partir de Davis y Wehmeyer (I99I). 


\begin{tabular}{|c|c|c|}
\hline Material y objetivo(s) & Elementos que trabajan & Aspectos metodológicos \\
\hline $\begin{array}{l}\text { Modelo de aprendizaje } \\
\text { autorregulado de Palmer y } \\
\text { Wehmeyer (2002) }\end{array}$ & \multirow{2}{*}{$\begin{array}{l}\text { - Roles de la familia en el apoyo } \\
\text { a la autodeterminación. } \\
\text { - Definición y expresión de } \\
\text { preferencias y metas del niño } \\
\text { con discapacidad. } \\
\text { - Estrategias de autorregulación. } \\
\text { - Competencias de } \\
\text { comunicación y autodefensa. }\end{array}$} & \multirow{2}{*}{$\begin{array}{l}\text { - Se trabaja en el ámbito } \\
\text { familiar. } \\
\text { - Consta de } 5 \text { capítulos, } \\
\text { presentados con un formato } \\
\text { sencillo y atractivo (con } \\
\text { ejemplos prácticos, tablas } \\
\text { explicativas y fichas de } \\
\text { trabajo). }\end{array}$} \\
\hline $\begin{array}{l}\text { - Capacitar a los padres para } \\
\text { apoyar a su hijo en la consecución } \\
\text { de metas escolares o personales. }\end{array}$ & & \\
\hline $\begin{array}{l}\text { Currículum familiar de Abery et al. } \\
\text { (1994) }\end{array}$ & \multirow{2}{*}{$\begin{array}{l}\text { - } \quad \text { Concepto de } \\
\text { - } \text { Plan de futuro de la persona } \\
\text { con discapacidad. } \\
\text { - Toma de decisiones familiares. } \\
\text { - Valores y metas. } \\
\text { - Elecciones y decisiones. } \\
\text { - Resolución de problemas y } \\
\text { - Conflictos interpersonales. } \\
\text { - } \text { de la comuniento de los recursos } \\
\text { - Habilidades de autodefensa } \\
\text { (por ejemplo, participación en } \\
\text { el sistema político). }\end{array}$} & \multirow{2}{*}{$\begin{array}{l}\text { - Consta de } 15 \text { módulos ( } 30 \\
\text { horas de duración). } \\
\text { - La familia cuenta con la ayuda } \\
\text { de un profesional (facilitador). } \\
\text { - Participa activamente la } \\
\text { persona con discapacidad, } \\
\text { como un miembro más de la } \\
\text { familia. }\end{array}$} \\
\hline $\begin{array}{l}\text { Dotar a las familias de las } \\
\text { destrezas necesarias para apoyar } \\
\text { a la persona con discapacidad en } \\
\text { el ejercicio de sus habilidades de } \\
\text { autodeterminación. } \\
\text { - Promover que todos los miembros } \\
\text { de la familia adquieran mayor } \\
\text { control sobre sus vidas. }\end{array}$ & & \\
\hline $\begin{array}{l}\text { Manual de formación para familias } \\
\text { de Ponce (2010) }\end{array}$ & \multirow{2}{*}{ 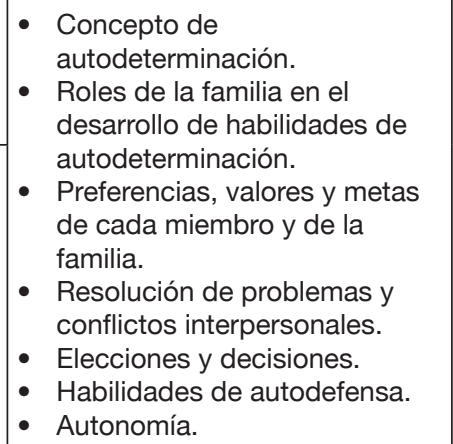 } & \multirow{2}{*}{$\begin{array}{l}\text { - Consta de } 10 \text { módulos (30 } \\
\text { horas de duración), cada } \\
\text { uno de los cuales tiene estas } \\
\text { secciones: presentación, } \\
\text { introducción teórica, } \\
\text { actividades, tareas para casa } \\
\text { y materiales adicionales. } \\
\text { - Guía a la familia un facilitador. } \\
\text { - Basado en Abery et al. (1994). }\end{array}$} \\
\hline $\begin{array}{l}\text { Sensibilizar a las familias } \\
\text { de jóvenes y adultos con } \\
\text { discapacidad intelectual sobre el } \\
\text { concepto de autodeterminación } \\
\text { y sobre algunos de los elementos } \\
\text { que lo integran. }\end{array}$ & & \\
\hline
\end{tabular}

Fuente: Elaboración propia a partir de Palmer y Wehmeyer (2002), Abery et al. (I994) y Ponce (2010).

discapacidad intelectual. En este artículo, se ha realizado una descripción de los factores que han contribuido a gestar y reforzar este movimiento de autodeterminación, así como de aquellas cuestiones elementales que permiten comprender mejor este concepto. Además, se han aportado sugerencias y propuestas de evaluación e intervención psicoeducativa, tanto del panorama internacional como de nuestro país.

Entendemos que son varios los agentes facilitadores de autodeterminación y que el logro de este derecho es una tarea compartida por todos. No queda sino plantear aquellos retos y desafíos que, a nuestro modo de ver, deben afrontarse durante los próximos años. A continuación, detallamos algunas de esas tareas, por ámbitos de actuación.

\subsection{Profesionales e investigadores}

Uno de los retos más importantes es desarrollar procesos de investigación-acción en los que 
teoría y práctica vayan de la mano. El objetivo es poner de manifiesto la relación existente entre el ejercicio de la autodeterminación y la consecución por parte de las personas con discapacidad de resultados positivos, como por ejemplo, la autonomía personal, la vida independiente, el acceso al empleo, la salud y bienestar psicológico o la calidad de vida. Los profesionales, como representantes y figuras de apoyo esenciales, tienen un papel fundamental a la hora de difundir la cultura de autodeterminación y favorecer la participación de estas personas en la comunidad. Debemos seguir generando espacios inclusivos que nos permitan convivir y aprender de la diversidad, así como crear entornos verdaderamente accesibles. Algunos campos de interés para la investigación de los próximos años son la formación de los profesionales y familias, o la validación de programas para la promoción de este derecho a lo largo de toda la vida de la persona.

\subsection{Familias}

La investigación actual da cuenta de la necesidad de llevar a cabo proyectos de formación específicamente dirigidos a las familias que, todavía hoy, encuentran muchas dificultades a la hora de apoyar en esta área a sus hijos con discapacidad. Así pues, una de las líneas prioritarias de actuación es la implicación de las familias en este proceso de cambio, que supone guiarlas desde el paradigma de la protección (en el que todavía se mueven muchas) hacia el paradigma de la autodeterminación. Para ello, se pueden tomar como referencia las siguientes orientaciones: proporcionar pautas que se integren en las rutinas y dinámicas familiares, equilibrar calidad de vida familiar e individual, promover enfoques de intervención colaborativos y centrados en la familia para impulsar la autodeterminación, o diseñar y validar programas de formación.

\subsection{Personas con discapacidad}

Para finalizar, ante todo la autodeterminación es un derecho que debe ser ejercido y defendido por las personas con discapacidad intelectual y asegurado por la sociedad en su conjunto. En ese sentido, son muchos los esfuerzos que se están realizando para trabajar no sólo para las personas con discapacidad, y en su nombre, sino junto a ellas. El propio Manifiesto por la plena ciudadanía... de este colectivo (mencionado en este texto) y las diversas iniciativas para mejorar sus habilidades de autodefensa y autogestión son buena muestra de ello. Es preciso prestar atención a grupos especialmente vulnerables, dentro del propio colectivo; concretamente, a los más pequeños y a aquellos con discapacidades severas o graves. Ello requiere repensar la autodeterminación y ahondar en este concepto como una sana interdependencia, enfatizar el papel de la persona de apoyo o aprovechar las posibilidades que brindan las nuevas tecnologías para facilitar la expresión de preferencias.

Trabajar a favor de la autodeterminación implica fundamentalmente un cambio de perspectiva. Supone considerar a las personas con discapacidad como capaces de participar en el desarrollo de su propia vida, y verlas como sujetos con derechos, talentos, habilidades, fortalezas y necesidades de apoyos. Nos exige, además, dejar de pensar en programas a los que las personas con discapacidad tienen que adaptarse y preguntarles por lo que ellas quieren. En definitiva, sustituir el control externo y experto, que lleva a la dependencia, por la capacitación de la persona (empowerment), que lleva a la autodeterminación. 
Abery, B. y Stancliffe, R.J. (2003a): “A tripartiteecological theory of self-determination”, en Wehmeyer, M.L. et al. (coords.), Theory in Selfdetermination. Foundations for Educational Practice, Springfield: Charles C. Thomas.

- (2003b): "An ecological theory of selfdetermination: Theoretical foundations", en Wehmeyer, M.L. et al. (coords.), Theory in Selfdetermination. Foundations for Educational Practice, Springfield: Charles C. Thomas.

Abery, B. y Zajac, R. (I996): "Self-determination as a goal of early childhood and elementary education", en Sands, D.J. y Wehmeyer, M.L. (coords.), Self-determination across the Life Span. Independence and Choice for People with Disabilities, Baltimore: Paul H. Brookes Publishing.

Abery, B., et al. (2000): Minnesota Selfdetermination Scales, Minnesota: Institute on Community Integration of the University of Minnesota.

Abery, B., et al. (I995): "Evaluating a multicomponent program for enhancing the self-determination of youth with disabilities", Intervention in School and Clinic, 30 (3): I70I79.

Abery, B. et al. (I994): Self-determination for Youth with Disabilities. A Family Education Curriculum, Minnesota: Institute on Community Integration of the University of Minnesota.

Agran, M., et al. (2008): "Promoting student active classroom participation skills through instruction to promote self-regulated learning and self-determination", Career Development for Exceptional Individuals, 3 I, IO6-I I4.

Algozzine, B., et al. (200I): "Effects on interventions to promote self-determination for individuals with disabilities", Review of Educational Research, 7I (2): 219-277.

American Association on Intellectual and Developmental Disabilities (2010): Intellectual
Disability: Definition, Classification and Systems of Support, I I ${ }^{\text {a }}$ ed., Washington, D.C.: American Association on Intellectual and Developmental Disabilities.

Arellano, A. (20I2): "El contexto familiar como apoyo a la autodeterminación de las personas con discapacidad intelectual. Estudio sobre sus cualidades según un enfoque centrado en la familia" [tesis doctoral inédita], Pamplona: Universidad de Navarra.

Arteta, R. et al. (2012): Planificación centrada en la persona. Una guía práctica (en línea), <http:// issuu.com/anfasnavarra/doc $>$, acceso 3 de noviembre de $20 \mathrm{I} 2$.

ASPRONA (2002): La autodeterminación de las personas con discapacidad intelectual, Madrid: FEAPS.

AZTEGUI (2009): Autodeterminación. Guia para el personal de apoyo, Madrid: FEAPS.

Brotherson, M.J., et al. (2008): “Understanding self-determination and families of young children with disabilities in home environments", Journal of Early Intervention, 3I (I): 22-43.

Brown, I., et al. (2007): "Person-centered and family-centered supports”, en Brown, I. y Percy M. (coords.), A Comprehensive Guide to Intellectual and Developmental Disabilities, Baltimore: Paul H. Brookes.

Calkins, C. et al. (20I I): "Introduction to the special issue on scaling up efforts to promote the self-determination of people with developmental disabilities”, Exceptionality. A Special Education Journal, I9 (I): 2-5.

Chambers, C.R., et al. (2007): "Self-determination: What do we know? Where do we go?", Exceptionality, I 5 (I): 3-I 5 .

Cobb, B., et al. (2009): "Self-determination for students with disabilities: A narrative metasynthesis", Career Development for Exceptional Individuals, 32 (2): I08-I I4. 
Confederación Española de Organizaciones a favor de las Personas con Discapacidad Intelectual (20I2): Manifiesto por la plena ciudadanía de las personas con discapacidad intelectual (en línea), <http://www.feaps.org/files/Manifiesto_ Ciudadania_I9-IO-I2.pdf $>$, acceso $30 \mathrm{de}$ octubre de $20 \mathrm{I} 2$.

Cook, C.C., et al. (2007): "Children with disabilities: Opportunities in the home environment", Implications, 5 (I 2): I-6.

Davis, S. y Wehmeyer, M.L. (I99I): Ten Steps to Independence: Promoting Self-Determination in the Home, Arlington: The Arc.

Deci, E.L. y Chandler, C.L. (I986): “The importance of motivation for the future of the LD field", Journal of Learning Disabilites, 23 (I): 587-594.

Doss, B. y Hatcher, B. (I996): "Self-determination as a family affair: Parents' perspectives on selfdetermination", en Sands, D.J. y Wehmeyer, M.L. (coords.), Self-determination across the Life Span. Independence and Choice for People with Disabilities, Baltimore: Paul H. Brookes Publishing.

Dykens, E. (2006): “Toward a positive psychology of mental retardation", American Journal of Orthopsychiatry, 76 (2): 18 5-193.

Erwin, E.J., et al. (2009): "How to promote self-determination for young children with disabilities: Evidenced-based strategies for early childhood practitioners and families", Young Exceptional Children, I2 (2): 27-37.

Fetterman, D.M. (I996): "Empowerment evaluation: An introduction to theory and practice”, en Fetterman, D.M. et al. (coords.), Empowerment Evaluation. Knowlegde and Tools for Self-assessment and Accountability, Thousand Oaks: Sage Publications.

Field, S. y Hoffman, A. (2002): "Preparing youth to exercise self-determination: Quality indicators of school environments that promote the acquisition of knowledge, skills and beliefs related to self-determination", Journal of Disability Policy Studies, I 3 (2): II4-II9.
- (1996): Steps to Self-determination: A Curriculum to Help Adolescents Learn to Achieve their Goals. Instructor's Guide, Austin: Pro-Ed.

Field, S., et al. (I998a): A Practical Guide for Teaching Self-determination, Reston: The Council for Exceptional Children.

Field, S., et al. (I998b): Self-determination Strategies for Adolescents in Transition, Austin: Pro-Ed.

Finlay, W. y Lyons, E. (200I): “Methodological issues in interviewing and using self-report questionnaires with people with mental retardation”, Psychological Assessment, I3 (3): 319-335.

Gómez-Vela, M., et al. (2OI2): “Assessment of the self-determination of Spanish students with intellectual disabilities and other educational needs", Education and Training in Autism and Developmental Disabilities, 47 (I): 48-57.

Heller, T., et al. (20I I): "Self-determination across the life span: Issues and gaps", Exceptionality. A Special Education Journal, I9 (I): 3 I-45.

Hoffman, A. y Field, S. (2006): Steps to Selfdetermination. A Curriculum to Help Adolescents Learn to Achieve their Goals, $2^{\mathrm{a}}$ ed., Austin: Pro-Ed.

Hoffman, A., et al. (2004a): Self-determination Assessment Battery User's Guide, $2^{\mathrm{a}}$ ed., Detroit: Wayne State University.

Hoffman, A. et al. (2004b): Self-Determination Assessment Battery User's Guide, $3^{\mathrm{a}}$ ed., Detroit: Center for Self-Determination and Transition, Wayne State University.

Illán, N. et al. (2005): Lo que dicen las familias. I 2 claves para la autonomía de las personas con sindrome de Down, Madrid: FEISD; Obra Social Caja Madrid.

Karvonen, M., et al. (2004): "Putting selfdetermination into practice", Exceptional Children, 7I (I): 23-4I.

Kim, K. y Turnbull, A. (2004): "Transition to adulthood for students with severe intellectual disabilities: Shifting toward person-family 
interdependent planning", Research and Practice for Persons with Severe Disabilities, 29 (I): 53-57.

Leal, L. (1999): Un enfoque de la discapacidad centrado en la familia, Madrid: Confederación Española de Organizaciones en favor de las Personas con Discapacidad Intelectual.

Lee, Y. et al. (2012): "Examining individual and instruction-related predictors of the selfdetermination of students with disabilities: Multiple regression analyses", Remedial and Special Education, 33 (3): I 50-I6I.

Linley, P.A., et al. (2006): "Positive psychology: Past, present, and (possible) future", The Journal of Positive Psychology, I (I): 3-I6.

Malik, P.B., et al. (I99I): "Interviewing young adults with mental retardation: A seldom used research method", Therapeutic Recreation Journal, 25: 60-73.

Martin, J.E. y Marshall, L.H. (I997): “Choice making: Description of a model project”, en Agran, M. (coord.), Student Directed Learning. Teaching Self-determination Skills, Pacific Grove: Brooks/Cole Publishing Company.

- (I996): “ChoiceMaker: Infusing selfdetermination instruction into the IEP and transition process", en Sands, D. J. y Wehmeyer, M. L. (coords.), Self-determination across the Life Span: Independence and Choice for People with Disabilities. Baltimore: Brookes.

Matuszewski, J. (1996): "Helping Tom learn to do for himself... with help", en Powers, L. et al. (coords.), Promoting Self-competence in Children and Youth with Disabilities. On the Road to Autonomy, Baltimore: Paul H. Brookes Publishing.

Mithaug, D.E. (2003): “Adjusting beliefs about self-determination", en Wehmeyer, M.L. et al. (coords.), Theory in Self-determination. Foundations for Educational Practice, Springfield: Charles C Thomas Publisher, 249260.

Mithaug, D.E., et al. (1998): “The Self-determined Learning Model of Instruction. Engaging students to solve their learning problems", en Wehmeyer, M.L. y Sands, D.J. (coords.), Making it Happen. Student Involvement in Education Planning, Decision Making and Instruction, Baltimore: Paul H. Brookes Publishing.

Nisbet, J. (2000): “Transformando los sistemas de servicios educativos y humanos hacia la autodeterminación: la experiencia de New Hampshire”, Siglo Cero, I92 (6): 5 I-58.

Nota, L., et al. (2007): "Self-determination, social abilities and the quality of life of people with intellectual disability", Journal of Intellectual Disability Research, 5 I (I I): 850-865.

OMS (200I): Clasificación Internacional del Funcionamiento, de la Discapacidad y de la Salud, Madrid: Imserso.

Palmer, S.B. y Wehmeyer, M.L. (2002): A Parent's Guide to the Self-determined Learning Model for Early Elementary Students, Kansas: Beach Center on Disability.

Peralta, F. (20II): “Taller para mejorar la autodeterminación de los hermanos", en Lizasoáin, O. (coord.), Hermanos de personas con discapacidad intelectual. Guí para el análisis de necesidades y propuestas de apoyo, Logroño: Siníndice.

- (2008): "Educar en autodeterminación: profesores y padres como principales agentes educativos", Educación y Diversidad. Anuario Internacional de Investigación sobre Discapacidad e Interculturalidad, 2: I 5 I-I66.

- (2006): "La evaluación de la conducta autodeterminada desde la perspectiva del empowerment evaluation: revisión de instrumentos de medida", en Peralta, M.C. et al. (coords.), Podemos hacer oír su voz: claves para promover la conducta autodeterminada, Málaga: Aljibe.

Peralta, F. y Arellano, A. (2010): "Familia y discapacidad. Una perspectiva teórico-aplicada del Enfoque Centrado en la Familia para promover la autodeterminación”, Electronic Journal of Research in Educational Psychology, $8(3):$ I.339-I.362. 
Peralta, F., et al. (coords.) (2006): Podemos hacer oír su voz: claves para promover la conducta autodeterminada, Málaga: Ediciones Aljibe.

Peralta, F., et al. (2005): “Creencias y conocimientos de los profesores acerca de la conducta autodeterminada en personas con discapacidad cognitiva", Revista de Investigación Educativa, 23 (2): 433-448.

Ponce, A. (2010): Formación en autodeterminación para familias, Madrid: Confederación Española de Organizaciones en favor de las Personas con Discapacidad Intelectual.

Poston, D., et al. (2003): "A qualitative study on family quality of life", Mental Retardation, 4I (5): 3I $3-328$.

Powers, L.E., et al. (I996). "Take Charge: A model for promoting self-determination among adolescents with challenges", en Powers, L.E. et al. (coords.), Promoting Self-competence in Children and Youth with Disabilities, Baltimore: Paul H. Brookes.

Ryan, R.M. y Deci, E.L. (coords.) (200I): Handbook of Self-determination Research, Rochester: University of Rochester Press.

Schalock, R.L. (2006): "La calidad de vida como agente del cambio: oportunidades y retos”, en Verdugo, M.A. y Jordán de Urríes, F.B. (coords.), Rompiendo inercias. Claves para avanzar. VI Jornadas Cientificas de Investigación sobre Personas con Discapacidad, Salamanca: Amarú Ediciones, I 5-39.

- (2000): "Three decades of quality of life", en Wehmeyer, M.L. y Patton, J.R. (coords.), Mental Retardation in the $2 I^{\text {st }}$ century, Austin, Texas: Pro-Ed.

Schalock, R.L. y Verdugo, M.A. (2010): Calidad de vida: Manual para profesionales de la educación, salud y servicios sociales, Madrid: Alianza.

Schalock, R.L., et al., (2010): "Quality of life model development and use in the field of intellectual disability”, en Kober R. (coord.), Enhancing the Quality of Life of People with Intellectual Disabilities, New York: Springer.
Schalock, R.L. et al. (2005): "Cross-cultural study of quality of life indicators", American Journal on Mental Retardation, I IO (4), 298-3 I I.

Schalock, R.L., et al. (2002): "Conceptualization, measurement, and application of quality of life for persons with intellectual disabilities: Report of an international panel of experts", Mental Retardation, 40, 457-470.

Seligman, M.E.P. y Csikszentmihalyi, M. (2000): "Positive psychology: An introduction", American Psychologist, 55 (I): 5-I4.

Turnbull, A.P. y Turnbull, H.R. (200I): "Selfdetermination for individuals with significant cognitive disabilities and their families", Association for Persons with Severe Handicaps, 26 (I): 56-62.

- (2000): "Fostering family-professional partnerships”, en Snell, M.E. y Brown, F. (coords.), Instruction of Students with Severe Disabilities, $5^{\text {a }}$ ed., New Jersey: Prentice Hall.

- (I996): "Group action planning as a strategy for providing comprehensive family support”, en Koegel, L.K. y Dunlap, G. (coords.), Positive Behavioral Support: Including People with Difficult Behavior in the Community, Baltimore: Brookes Publishing Company.

Turnbull, A.P., et al. (2006): Families, Professionals, and Exceptionality: Positive Outcomes through Partnerships and Trust, $5^{\mathrm{a}}$ ed., New Jersey: Pearson/Merrill- Prentice Hall.

Verdugo, M.A. (ed.). (2006): Cómo mejorar la calidad de vida de las personas con discapacidad: instrumentos y estrategias de evaluación, Salamanca: Amarú.

Verdugo et al. (2009): Escala Integral. Evaluación objetiva y subjetiva de la calidad de vida, Madrid: CEPE.

Walker, H.M., et al. (20 I I): “A socio-ecological approach to promote self-determination", Exceptionality. A Special Education Journal, I9 (I), 6-I 8 .

Ward, M.J. y Kohler, P.D. (I996): “Teaching selfdetermination content and process", en Powers, L.E. et al. (coords.), Promoting Self-competence 
in Children and Youth with Disabilities, Baltimore: Paul H. Brookes Publishing.

Wehmeyer, M.L. (2009): “Autodeterminación y la tercera generación de prácticas de inclusión”, Revista de Educación, 349: 45-67.

- (2005): "Self-determination and individuals with severe disabilities: Re-examining meanings and misinterpretations", Research and Practice for Persons with Severe Disabilities, 30 (3): I I3I2O.

- (2004): "Beyond self-determination: Causal agency theory", Journal of Developmental and Physical Disabilities, I6 (4), 337-359.

- (I999): “A functional model of selfdetermination: describing development and implementing instruction", Focus on Autism and Other Developmental Disabilities, I4 (I): 53-6I.

- (I998): "Self-determination and individuals with significant disabilities: Examining meanings and misinterpretations", Journal of the Association for Persons with Severe Handicaps, 23 (I): 5-I 6.

- (I995): The Arc's Self-determination Scale. Procedural Guidelines, Arlington: The Arc of the United States.

Wehmeyer, M.L. y Field, S. (2007): Selfdetermination: Instructional and Assessment Strategies, Thousand: Corwin Press.

Wehmeyer, M.L. y Ward, M.J. (I995): “The spirit of the IDEA mandate: Student involvement in transition planning", Journal of the Association for Vocational Special Needs Education, I7: IO8-III.

Wehmeyer, M.L., et al. (2OI I): "Personal selfdetermination and moderating variables that impact efforts to promote self-determination", Exceptionality. A Special Education Journal, I9 (I), I9-30.

Wehmeyer, M.L., et al. (2009): "Selfdetermination”, en López, S.J. y Snyder, C.R. (coords.), Oxford Handbook of Positive

Psychology, $2^{\text {a }}$ ed., New York: Oxford

University Press.

Wehmeyer, M.L., et al. (2008): "Self-determination and students with developmental disabilities", en Parette, H.P. y Peterson-Karlan, G.R. (coords.), Research-based Practices in Developmental Disabilities, $2^{\mathrm{a}}$ ed., Texas: Pro Ed.

Wehmeyer, M.L., et al. (2006): Escala de autodeterminación ARC. Adaptación española de las versiones para adolescentes y adultos, Madrid: CEPE.

Wehmeyer, M.L., et al. (2002): Teaching Students with Mental Retardation. Providing Access to the General Curriculum, Baltimore: Paul H. Brookes Publishing.

Wehmeyer, M.L., et al. (2000a): "Promoting causal agency: The self-determined learning model of instruction", Exceptional Children, 66, 430453.

Wehmeyer, M.L., et al. (2000b): "Riding the third wave: Self-determination and self-advocacy in the $2 \mathrm{I}^{\mathrm{st}}$ century", Focus in Autism and Other Developmental Disabilities, I 5 (2): I06-I I 5.

Wehmeyer, M.L., et al. (1998): A Teacher's Guide to Implementing the Self-determined Learning Model of Instruction. Adolescent Version, Arlington: The Arc.

Wolman, J.M., et al. (I994): AIR Selfdetermination Scale and User Guide, Palo Alto: American Institutes for Research.

Wood, W.M. et al. (2005): "A review of selfdetermination interventions with individuals with severe disabilities", Research and Practice for Persons with Severe Disabilities, 30 (3): I 2 I-I 46 .

Zulueta, A. y Peralta, F. (2008): "Percepciones de los padres acerca de la conducta autodeterminada de sus hijos con discapacidad intelectual", Siglo Cero. Revista Española sobre Discapacidad Intelectual, 39 (I): 3 I-43. 